\title{
Ermittlung von Koronargefäßverläufen in 3D-Kontrastechokardiogrammen
}

\author{
Uwe Graichen $^{1}$, Rainer Zotz ${ }^{2}$, Philipp Wild ${ }^{2}$ und Dietmar Saupe ${ }^{1}$ \\ 1 Institut für Informatik, Universität Leipzig \\ 04109 Leipzig, Augustusplatz 10 - 11 \\ 2 Herzzentrum, Universität Leipzig \\ 04289 Leipzig, Russenstr. 19 \\ zotr@medizin.uni-leipzig.de
}

\begin{abstract}
Zusammenfassung Im vorliegenden Artikel wird ein Volumerenderingverfahren vorgestellt, das für Visualisierung von stark gerichteten, röhrenförmigen Strukturen konzipert ist. In einem Analyseschritt wird mit Hilfe von Strukturtensoren für jedes Voxel des Volumens ein Maß für Kohärenz (Ähnlichkeit mit einer Röhre) ermittelt. In Abhängigkeit dieses Maßes wird der Transparenzwert der Voxel gesetzt. Das Visualisierungsverfahren wird für die Darstellung der Verläufe von Koronarien in 3D-Kontrastechokardiogrammen verwendet.
\end{abstract}

\section{Einleitung}

Haupttodesursache in den westlichen Ländern sind koronare Herzerkrankungen. Momentan ist die Röntgenangiographie Goldstandard und einziges Verfahren bei der Beurteilung der Koronargefäße. Die Röntgenangiographie ist ein invasives, röntgenbasiertes Verfahren, das der Infrastruktur eines Herzkathederlabors bedarf. Das Untersuchungsverfahren ist mit einer Belastung durch ionisierende Strahlung für Patient und untersuchenden Arzt verbunden und kann nicht beliebig oft wiederholt werden.

Ultraschall gewinnt in der Kardiographie als bildgebendes Verfahren zunehmend an Bedeutung. Im Gegensatz zu dem auf Röntgen basierenden Verfahren erfolgt keine Belastung des Patienten und des Arztes durch ionisierende Strahlung. Echogeräte sind, verglichen mit anderen bildgebenden Geräten, preiswert und sehr stark verbreitet.

Mit dem hier vorgestellten Verfahren können röhrenförmige Strukturen dargestellt werden. Das Verfahren wird mit Ultraschalldatensätzen verwendet. Texturen in Ultraschallaufnahmen enthalten wichtige Informationen für die Befundung. Sie werden bei diesem Visualisierungsverfahren mit dargestellt.

\section{Strukturanalyse des Datensatzes und Bestimmung des Kohärenzmaßes}

\subsection{Strukturtensor}

Die lokale Struktur eines Volumendatensatz $u(x)$ kann mittels Strukturtensoren [1-3] analysiert werden. Ein Volumendatensatz $u(x)$ ist eine Abbildung $u: \Omega \rightarrow \mathbb{R}, \Omega$ ist 
eine dreidimensionale Domäne $\Omega=\left(0, a_{1}\right) \times\left(0, a_{2}\right) \times\left(0, a_{3}\right)$. Das Volumen $u(x)$ wird mit einem Gaußkern $K_{\sigma}$ der Standardabweichung $\sigma$ gefaltet.

$$
u_{\sigma}(x)=K_{\sigma} * u(x)
$$

Dadurch werden Störungen in der Größenordnung $\sigma$ aus dem Volumen entfernt. Aus dem geglätteten Volumen $u_{\sigma}(x)$ werden die Gradienten $\nabla u_{\sigma}(x)$ berechnet. Bildet man aus den Gradienten $\nabla u_{\sigma}(x)$ das Tensorprodukt, erhält man eine symmetrische, positiv semidefinite Matrix.

$$
J\left(\nabla u_{\sigma}(x)\right)=\nabla u_{\sigma}(x) \otimes \nabla u_{\sigma}(x)=\nabla u_{\sigma}(x) \nabla u_{\sigma}(x)^{T}
$$

Den Strukturtensor $J_{\rho}\left(\nabla u_{\sigma}\right)$ erhält man durch komponentenweise Faltung von $J\left(\nabla u_{\sigma}\right)$ mit einem Gaußkern $K_{\rho}$ der Standardabweichung $\rho$.

$$
J_{\rho}\left(\nabla u_{\sigma}\right)=K_{\rho} * J\left(\nabla u_{\sigma}\right)
$$

Der Parameter $\rho$ ist ein Skalenparameter, mit dem der Durchmesser der Strukturen angegeben werden kann, die dargestellt werden sollen. Die Eigenwerte $\mu_{1}, \mu_{2}, \mu_{3}$ des Strukturtensors $J_{\rho}\left(\nabla u_{\sigma}\right)$ mit $\mu_{1} \geq \mu_{2} \geq \mu_{3}$ entsprechen den Kontrasten in Richtung der Hauptorientierungen. Die zugehörigen Eigenvektoren $\boldsymbol{v}_{\mathbf{1}}, \boldsymbol{v}_{\mathbf{2}}, \boldsymbol{v}_{\mathbf{3}}$ weisen in Richtung der lokalen Hauptorientierung, siehe Abbildung 1(a). Der Vektor $\boldsymbol{v}_{1}$ verläuft parallel zum Gradienten, $\boldsymbol{v}_{\mathbf{3}}$ weist in die Kohärenzrichtung (orthogonal zu $\boldsymbol{v}_{\mathbf{1}}$ ) und $\boldsymbol{v}_{\mathbf{2}}$ steht senkrecht auf $v_{1}$ und $v_{3}$.

\subsection{Das Maß für Kohärenz}

Das Maß für die Kohährenz $\tau$ wird aus den Eigenwerten $\mu_{1}, \mu_{2}, \mu_{3}$ des Strukturtensors, mit $\mu_{1}, \mu_{2}, \mu_{3} \in\left(0, \mu_{\max }\right)$ und $\mu_{1} \geq \mu_{2} \geq \mu_{3}$, berechnet. Alle möglichen Wertekombinationen $\left(\mu_{1}, \mu_{2}, \mu_{3}\right)$ werden von einem Tetraeder mit den Eckpunkten $(0,0,0)$, $\left(\mu_{\max }, 0,0\right),\left(\mu_{\max }, \mu_{\max }, 0\right)$ und $\left(\mu_{\max }, \mu_{\max }, \mu_{\max }\right)$ (Abbildung $\left.1(\mathrm{~b})\right)$ eingegrenzt. Die einzelnen Eckpunkte haben dabei folgende Bedeutung:

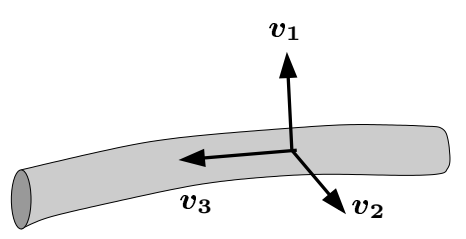

(a) Strukturtensor

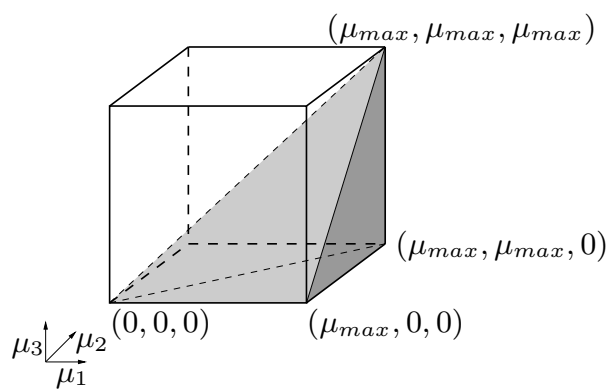

(b) Kohärenzmaß

Abbildung 1. (a) Strukturtensor an einer kohärenten Struktur, (b) Ermittlung des Kohärenzmaßes aus den Eigenwerten des Strukturtensors 
- $(0,0,0)$ - kontrastloser Punkt in Umgebung mit identischen Grauwerten

- $\left(\mu_{\max }, 0,0\right)$ - Punkt in Ebene, der maximalen Kontrast zur Umgebung aufweist

- $\left(\mu_{\max }, \mu_{\max }, 0\right)$ - Punkt in Struktur, mit maximaler Kohärenz

- $\left(\mu_{\max }, \mu_{\max }, \mu_{\max }\right)$ - Punkt mit maximalem Kontrast zur Umgebung

Die Punkte $(0,0,0),\left(\mu_{\max }, 0,0\right)$ und $\left(\mu_{\max }, \mu_{\max }, \mu_{\max }\right)$ beschreiben eine Ebene $E_{0}$, der das Kohärenzmaß $\tau=0$ zugeordnet wird. Die Ebene $E_{0}$ ist in Abbildung 1 hellgrau dargestellt. Als Kohärenzmaß $\tau$ für röhrenförmige Strukturen schlagen wir das Quadrat des Abstandes von der Ebene $E_{0}$ vor.

$$
\tau=\frac{1}{2}\left(\mu_{2}-\mu_{3}\right)^{2}
$$

\section{Implementation}

Der Schätzer für das Maß der Kohärenz ist als C++ Klasse implementiert und wird mit dem Volumerenderer der VTK Klassenbibliothek [4] verwendet. Die Transparenz der Voxel wird mit Hilfe einer Lookup-Table in Abhängigkeit vom Maß der Kohärenz gesetzt. Zum Testen wurde eine grafische Benutzerschnittstelle programmiert.

\section{Test des Verfahrens}

Das Verfahren wurde an einem künstlichen Datensatz und an Ultraschallvolumendatensätzen getestet.

\subsection{Künstlicher Datensatz}

Der künstliche Datensatz hat die Größe $272 \times 200 \times 76$ Voxel. Im Datensatz sind einige geometrische Gebilde angeordnet, Abbildung 2(a):

- drei Gruppen von je drei Tori mit $d=3,5,12$ und $D=20,60,100$

- drei Zylinder mit $d=60$ und $h=3,5,12$

- drei Zylinder mit $d=3,5,12$ und $h=50$

- drei Zylinder mit je zwei abzweigenden Zylindern $d=3,5,12$

- drei Kugelschalen mit $d=17,37,57$ und Schalenstärke je $s=3$

Den geometrischen Objekten im Testdatensatz wurde der Grauwert 120, dem Hintergrund der Grauwert 150 zugeordnet (Abbildung 2(a)). Zum Datensatz wurde ein additives, Gaußsches Rauschen mit Mittelwert 0 und einer Standardabweichung 10 hinzugefügt. In Abbildung 2(c) ist ein Schnittbild durch den künstlichen Volumendatensatz zu sehen. Die Antwort $\tau$ des Kohärenzmaßschätzers, mit den Parametern $\sigma=1$ und $\rho=4$, für dieses Schnittbild ist in Abbildung 2(d) dargestellt. Die röhrenförmigen Objekte mit $d=5$ (die Tori und der Zylinder) liefern eine starke Antwort. In Abbildung 2(b) ist der Datensatz volumegerendert dargestellt. Die Transparenz der Voxel wurde in Abhängigkeit vom Kohärenzma $\tau$ gesetzt. Das Kohärenzmaß wurde mit den Parametern $\sigma=1$ und $\rho=4$ ermittelt. Die röhrenförmige Objekte mit $d=5$ werden sehr gut 
dargestellt. Leichte Störungen werden durch die Kanten der Zylinder mit $d=60$ verursacht. Bei den Zylindern mit Abzweigungen, erhält man an den Abzweigungen nur eine sehr schwache Antwort. Für die Kugelschalen liefert der Kohärenzschätzer keine Antwort. Sie werden nicht mit abgebildet.

Der Datensatz wurde auf einem Rechner mit Pentium III Prozessor, 450 Mhz Taktfrequenz und 512 MByte Arbeitsspeicher in ca. 2.5 Minuten gerendert.

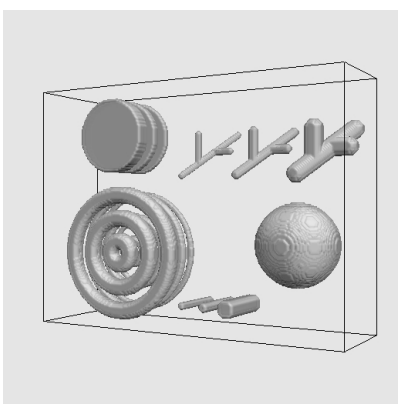

(a)

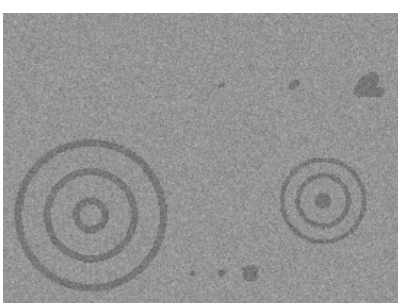

(c)

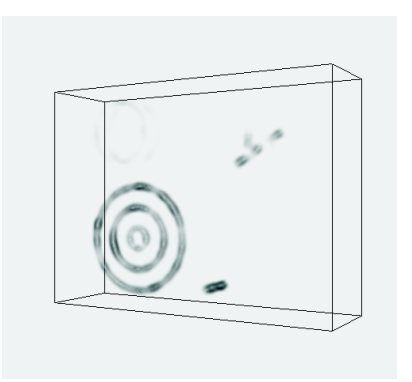

(b)

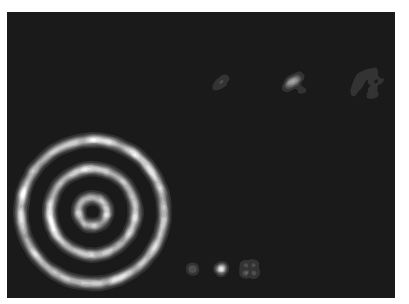

(d)

Abbildung 2. (a) Künstlicher Datensatz, (b) Volumegerenderter künstlicher Datensatz mit additiven Gaußschem Rauschen, (c) Schnitt durch künstlichen Datensatz mit additiven Gaußschem Rauschen, (d) Antwort des Kohärenzschätzers im Schnitt von (c)

\subsection{Ultraschalldatensatz}

Das Verfahren wurde auch an Ultraschallvolumendatensätzen getestet. Die Ultraschallbilder wurden mit einem HP Sonos 5500 und einer Omniplane TEE-Sonde (5Mhz) aufgenommen. Während der Aufnahme wird der Kopf der Sonde in $2^{\circ}$ Abständen gedreht. Die Aufzeichnung der 2D Einzelbilder erfolgt herzphasengetriggert. Die Volumendatensätze wurden aus den 2D Bildsequenzen mit Hilfe des Programms InVivoNT der Firma Medcom erstellt.

Mit Hilfe des vorgestellten Verfahrens ist es möglich, Gefäße abschnittsweise in Volumendatensätzen zu verfolgen. In der Abbildungen 3(a) ist ein Schnittbild durch einen Volumendatensatz abgebildet. In der Ecke links oben ist ein Gefäßstück zu sehen. Die Antwort des Kohärenzschätzers für dieses Schnittbild ist in der Abbildung 3(b) dargestellt. In Abbildung 3(c) ist ein volumegerenderter 3D-Ultraschalldatensatz zu sehen. 


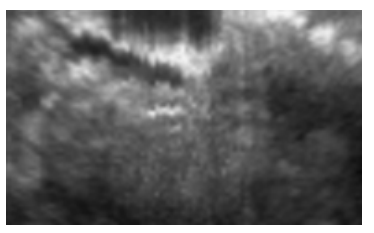

(a)

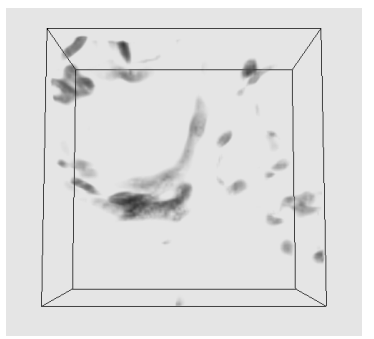

(c)

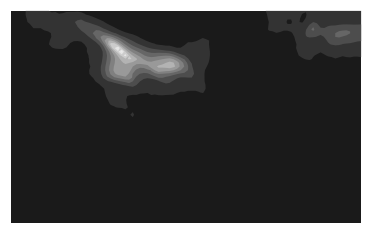

(b)

Abbildung 3. (a) Schnitt durch 3D-Ultrschalldatensatz, (b) Antwort des Kohärenzschätzers, (d) Volumegerenderter 3D-Ultraschalldatensatz

\section{Zusammenfassung und Ausblick}

Mit dem vorgestellten Verfahren lassen sich Gefäße in Ultraschallvolumendatensätzen abschnittsweise verfolgen und darstellen. Für die Darstellung der Gefäße werden die Grauwerte der Voxel des Ultraschalldatensatzes verwendet. Die Transparenz der Voxel wird in Abhängigkeit vom Kohärenzmaß gesetzt.

Das Verfahren kann verbessert werden durch die Verwendung eines Kohärenzschätzers der in der Lage ist, helle und dunkle röhrenartige Strukturen voneinander zu unterscheiden. Bei der Rekonstruktion der Volumendatensätzen aus den 2D-Einzelbildern wird davon ausgegangen, daß der Aufnahmekopf der Ultraschallsonde während der gesamten Aufnahmezeit um eine feste Achse rotiert. Bedingt durch die starke Eigenbewegung des Herzens und einer Aufnahmezeit von einigen Minuten ändert sich aber die Lage der Achse. Die Qualität der Volumendatensätze kann verbessert werden, indem die Ultraschalleinzelbilder vor der Rekonstruktion der Volumen registriert werden.

\section{Literatur}

1. J. Weickert. Coherence-enhancing diffusion filtering. International Journal of Computer Vision, 31(2/3):111-127, 1999.

2. J. Weickert. Anisotropic Diffusion in Image Processing. ECMI. Teubner, 1998.

3. B. Jähne. Spatio-temporal image processing: theory and scientific applications, volume 751 of Lecture Notes in Computer Science. Springer-Verlag, Berlin, Heidelberg, New York, 1993.

4. Will Schroeder, Ken Martin, and Bill Lorensen. The Visualization Toolkit: An Object-Oriented Approach to 3D Graphics. Prentice Hall Inc., 2nd edition edition, 1998. 